\title{
Etapas Prioritárias para uso de Dados Remotos e Sistemas de Informações Geográficas em Planejamento de Inventário, Avaliação e Monitoramento de Áreas Úmidas
}

Stela Rosa Amaral Gonçalves ${ }^{1}$, Cátia Nunes da Cunha ${ }^{1} \&$ Wolfgang Johannes Junk ${ }^{2}$

Recebido em 30/01/2018 - Aceito em 04/09/2019

RESUMO - A integração de dados remotos e de sistema de informação geográfica (SIG) tem a capacidade de fornecer informações, avaliar e monitorar sítios Ramsar e outras áreas úmidas (AUs) no tempo e no espaço. Assim, sabendo-se da necessidade que o Brasil possui de realizar, na prática, as diretrizes da Convenção Ramsar, são necessárias orientações para o planejamento de inventário, a avaliação e o monitoramento de AUs utilizando dados remotos e SIG. Neste trabalho, definiram-se passos integrados e orientações para garantir a realização de um inventário de AUs e atender às ações da atual Estratégia Nacional de Conservação e Uso Sustentável das Áreas Úmidas do Brasil.

Palavras-chave: Mapeamento de áreas úmidas; nível de mapeamento; SIG; sítios Ramsar.

\begin{abstract}
Priority Steps for Using Remote Data and Geographic Information Systems in Wetland Inventory Planning, Assessment, and Monitoring. Remote data and geographic information system (GIS) integration has the ability to provide information, evaluate and monitor Ramsar sites and other wetlands (AUs) in time and space. Thus, knowing that Brazil has to carry out, in practice, the Ramsar Convention guidelines, guidelines for the planning of inventory, evaluation and monitoring of AUs using remote data and GIS are necessary. In this paper, integrated steps and guidelines were defined to ensure an inventory of UAs and to meet the actions of the current National Strategy for Conservation and Sustainable Use of Wetlands in Brazil.
\end{abstract}

Keywords: Mapping wetlands; mapping level; GIS; Ramsar sites.

RESUMEN - Pasos prioritarios para el uso de datos remotos y sistemas de información geográfica en la planificación, evaluación y monitoreo de inventarios de humedales. La integración remota de datos y sistemas de información geográfica (SIG) tiene la capacidad de proporcionar información, evaluar y monitorear sitios Ramsar y otros humedales (UA) en el tiempo y el espacio. Por lo tanto, sabiendo que Brasil tiene que cumplir, en la práctica, las directrices de la Convención de Ramsar, las directrices para la planificación del inventario, la evaluación y el monitoreo de las UA utilizando datos remotos y SIG son necesarias. En este documento, se definieron pasos y pautas integradas para garantizar un inventario de las UA y cumplir con las acciones de la actual Estrategia Nacional para la Conservación y el Uso Sostenible de los Humedales en Brasil.

Palabras clave: Mapeo de humedales; nível de mapeo; SIG; sitios Ramsar.

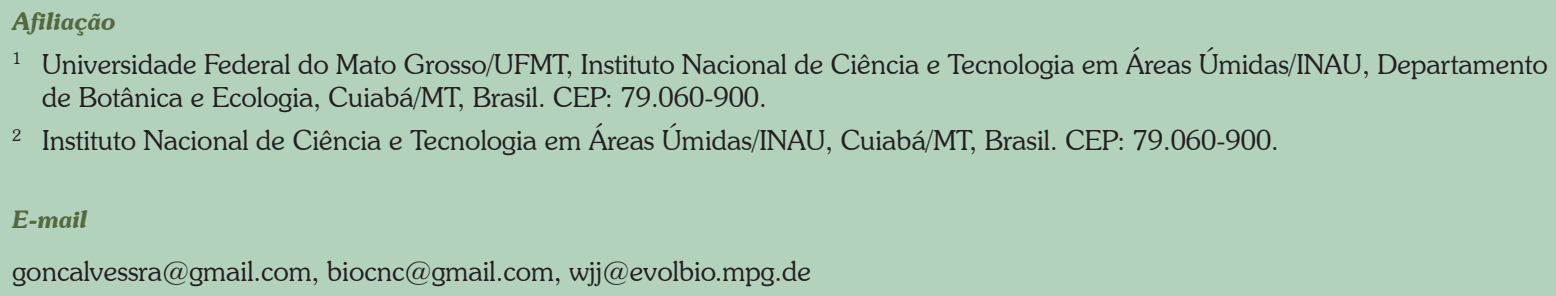




\section{Importância do uso do sensoriamento remoto e de sistemas de informações geográficas (SIGs) para o planejamento de inventário, avaliação e monitoramento de áreas úmidas (AUs)}

"O desenvolvimento de técnicas com uso de sensoriamento remoto e SIG para inventário, avaliação e monitoramento de AUs dos sítios Ramsar tem sido reconhecido na Convenção de Ramsar" (Davidson \& Finlayson 2007). Esse reconhecimento deu-se desde a Conferência das Partes (COP8), na qual as partes signatárias da Convenção Ramsar solicitaram a elaboração de orientações sobre aspectos contidos no inventário de AUs para a utilização de dados de sensoriamento remoto e SIG. Atendendo à demanda da $\mathrm{COP} 8$, foi formalizado um relatório técnico do secretariado da Convenção Ramsar e publicado em 2006 (Lowry 2006). Diversas informações referentes aos cuidados necessários para utilizar SIG e sensoriamento remoto como ferramenta em inventário, avaliação e monitoramento de AUs foram abordadas, como, por exemplo: os tipos de categorias de softwares, a natureza dos dados, questões relacionadas à escala, dentre outras orientações necessárias.

Em 2010, foi publicado o Manual 15 Ramsar de Inventário de Áreas Úmidas, que reconheceu a importância dos inventários para identificar sítios para a lista Ramsar, quantificar e avaliar o recurso global de AUs, identificar as AUs que devem ser restauradas, avaliar o risco e vulnerabilidade. Desta forma, dados remotos e SIG são ferramentas adequadas e reconhecidas para realização de inventário, avaliação e monitoramento de AUs, como, por exemplo: dados de fotografias aéreas, mapas topográficos e imagens de satélite.

"Dados de sensoriamento remoto e SIG integrados podem ser concebidos em diversas escalas espaciais e temporais, fornecendo informações sobre extensão, condições ambientais, informações de áreas de grande extensão, imagens de cobertura global de qualidade e resolução cada vez melhores, monitoramento das variações espaço-temporais e inúmeras possibilidades quando tratamos do mapeamento, monitoramento e gerenciamento dos recursos" (Rebelo et al. 2009). Para Nogueira (2008), essas ferramentas são um grande aliado para realizar análises espaciais para a tomada de decisões.

Apesar de ainda existirem projetos/trabalhos limitados pelo custo, tanto da aquisição de dados quanto do uso de softwares de SIG, existe uma tendência de diminuir essa dificuldade com o passar dos anos, visto que existem dados e softwares (visualizadores e desktop) de boa qualidade, disponíveis gratuitamente. Entretanto, muitas vezes os dados ou os softwares gratuitos podem não atender às necessidades específicas, como, por exemplo, de um inventário de pequenas AUs, sendo necessário adquirir dados com maior capacidade de resolução e escala compatível que sustentem todas as outras etapas e passos contidos em um planejamento de inventário de AUs utilizando dados remotos.

Atualmente, foi elaborada a Estratégia Nacional de Conservação e Uso Sustentável das Áreas Úmidas do Brasil, com objetivos relacionados à implantação e gestão de sítios Ramsar e incentivo a ações transversais para a conservação e o uso sustentável das áreas úmidas em todo o país, dentro ou fora de unidades de conservação. Assim, tendo em vista a importância do inventário para identificação dos sítios Ramsar e conservação das AUs, é de notável relevância definir questões-chave e etapas relacionadas às técnicas utilizando sensoriamento remoto e SIG. Assim, o inventário torna-se viável em escalas espacial e temporal, necessárias para identificar e avaliar as condições das AUs, tornando-se base para a tomada de decisões de órgãos públicos no país. Além de atender a uma demanda do objetivo da Estratégia Nacional de Conservação e Uso Sustentável das Áreas Úmidas do Brasil, o mapeamento dos aspectos ambientais e socioculturais das AUs e dos sítios Ramsar constitui-se em propostas de ações estratégicas a serem desenvolvidas pelo Ministério de Meio Ambiente e órgãos vinculados, Comitê Nacional de Zonas Úmidas (CNZU), gestores de sítios Ramsar e outras instâncias correlatas, para atender aos compromissos assumidos pelo Brasil perante a Convenção de Ramsar. 


\section{Etapas recomendadas para planejamento de um inventário, avaliação e monitoramento de AUs utilizando dados e/ou tecnologias remotas}

Baseando-se nos dados de Lowry (2006), nas etapas recomendadas por Ramsar (2010), nos Apêndices do Manual 15 e, no mais recente, Quadro Estruturado para o Planejamento de um Inventário de Áreas Úmidas via Sensoriamento Remoto e Geoprocessamento de Nunes (2017), foi possível estabelecer um fluxograma com etapas integradas e adaptadas a inventário, avaliação e monitoramento de AUs, realizado com dados remotos e softwares de SIG.

A primeira etapa (Figura 1), denominada definição do objetivo ou propósito do inventário, foi definida por Nunes (2017), adaptada do Manual 15 Ramsar (2010). Nessa etapa inicial, Nunes (2017) ressalta a necessidade de relacionar o objetivo do inventário, que pode ser com propósito ecológico, ambiental ou outros, com a escala e os dados a serem utilizados. Desta forma, partindo desse princípio, e levando em consideração que existem diversas bases de dados de sensoriamento remoto e SIG disponíveis, a segunda etapa consiste na busca pelos dados existentes da área de interesse, ou seja, um levantamento de dados secundários já existentes.

Nem todos os dados disponíveis podem ser utilizados no inventário. Por isso, após a busca pelos dados secundários, torna-se necessário realizar uma seleção do conjunto de dados que realmente atendam ao propósito do inventário, orientado sempre pela sua escala e resolução. De posse dos dados selecionados, deve-se escolher a categoria de software mais viável em que os dados se enquadram. O quarto e o quinto passos apresentados no fluxograma (Figura 1) definem questões-chave para a adoção de procedimentos comuns em planejamento de inventários, avaliações e monitoramento de AUs; assim, os potenciais usuários do SIG podem identificar quais são seus requisitos e capacidades.

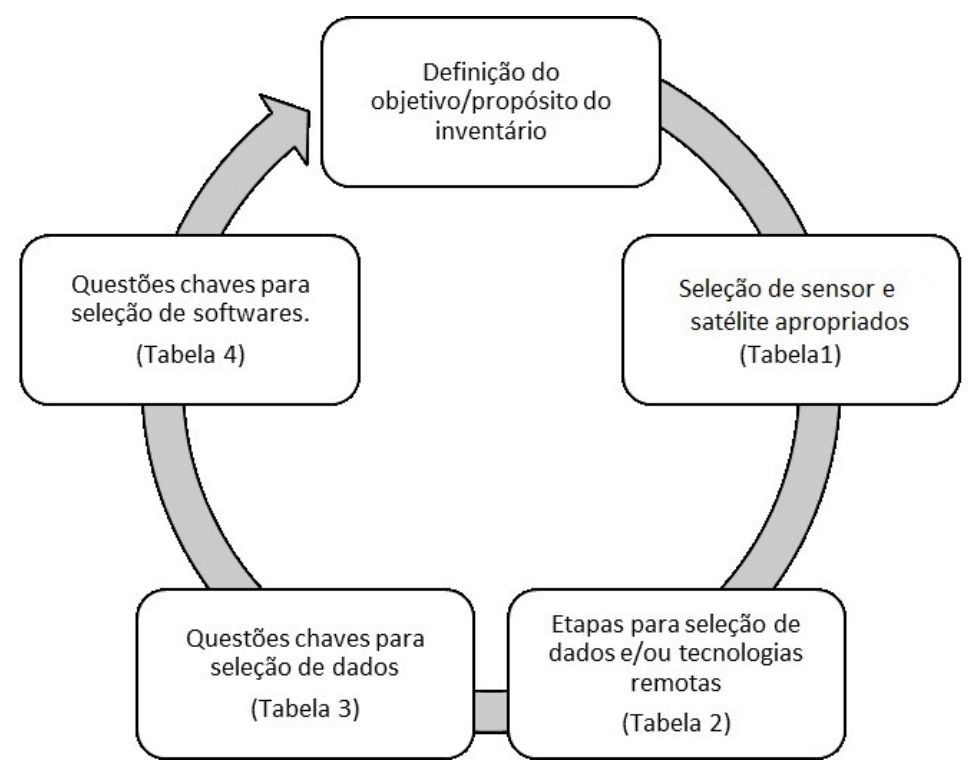

Figura 1 - Fluxograma de etapas recomendadas para o planejamento de inventário de áreas úmidas utilizando dados e tecnologias remotas. Fonte: Os autores.

Foram observadas quatro informações em comum na integração do Quadro Estruturado para o planejamento de um inventário de áreas úmidas do Manual 15 Ramsar (2010) e seu apêndice II, que trata de passos para determinação dos dados remotos mais apropriados para o inventário de AUs. As informações em comum contempladas foram: as relacionadas ao objetivo do inventário; escala/resolução dos dados; cronograma/amostragem em campo; e a viabilidade, 
que inclui o custo do suporte necessário para aquisição e processamento de dados SIG. Desta maneira, pode-se elencar essas informações como primordiais e de sustento a todas as outras contidas em um planejamento de inventário de AUs utilizando dados remotos. Nunes (2017) cita o objetivo e/ou propósito do inventário e a determinação da escala e a resolução explicitamente como passos a seguir no inventário; entretanto, trata de forma implícita as outras informações referentes ao levantamento em campo, à viabilidade da aquisição do software e ao processamento dos dados, ao nortear a escolha de um método adequado.

\section{Etapas apropriadas para a seleção de dados e/ou tecnologias remotas}

A determinação dos dados e das tecnologias obtidos remotamente, mais apropriados para inventário de AUs, segundo o apêndice II do Manual 15 Ramsar (2010) e Nunes (2017), foi simplificada na Tabela 2. A tabela fornece os passos para determinar os dados de detecção remota mais adequados, em seis etapas. Mais recentemente, Nunes (2017) cita diversos trabalhos que utilizaram sensores de média e alta resolução, e técnicas de geoprocessamento para mapear áreas úmidas no estado do Mato Grosso. Dentre as técnicas, citam-se: a classificação orientada a objeto (OBIA), o uso de índices espectrais (NDVI e MNDWI), a classificação com o algoritmo Support Vector Machine (SVM) e a utilização de produtos de veículos aéreos não tripulados (VANTs). Os principais sensores, suas resoluções e as respectivas bandas capazes de fornecer produtos gratuitos com média e grande escala para mapeamento de áreas úmidas foram listados na Tabela 1 . O CBERS 04, destacado na Tabela 1, tem previsão de ser lançado em órbita no segundo semestre deste ano, mas as características dos sensores já foram divulgadas no site do Instituto Nacional de Pesquisas Espaciais (INPE). Além dos citados na Tabela 1, ressalta-se que os outros satélites da família Landsat anteriores ao 8, segundo Nunes (2017), ainda são utilizados em mapeamentos de áreas úmidas. Satélites com alta resolução, como, por exemplo, o SPOT, IKONOS, PLANET e outros não foram inclusos por não fornecerem de forma gratuita suas imagens, restringindo o acesso apenas ao público com possibilidade de adquiri-los.

Tabela 1 - Sensores, resoluções e bandas espectrais de satélites potenciais para mapeamentos ambientais e de áreas úmidas. Fonte: Site oficial do INPE e Paranhos Filhos et al. (2016).

Table 1 - Sensors, resolutions and spectral bands of potential satellites for environmental and wetland mapping. Source: Official website INPE and Paranhos Filhos et al. (2016).

\begin{tabular}{|c|c|c|c|}
\hline Satélites & Sensores/câmeras & Resolução & Bandas \\
\hline \multirow{3}{*}{ CBERS 2B } & $\mathrm{CCD}$ & $20 \mathrm{~m}$ & $\begin{array}{c}\text { Azul, verde, vermelho, infravermelho } \\
\text { próximo, pancromática }\end{array}$ \\
\hline & HRC & $2,5 \mathrm{~m}$ & Pancromática \\
\hline & WFI & $260 \mathrm{~m}$ & Vermelho, infravermelho próximo \\
\hline \multirow{4}{*}{ CBERS 3 e 4} & PAN & $5 \mathrm{~m} / 10 \mathrm{~m}$ & $\begin{array}{l}\text { Pancromática, verde, vermelho, } \\
\text { infravermelho próximo }\end{array}$ \\
\hline & MUX & $20 \mathrm{~m}$ & $\begin{array}{l}\text { Azul, verde, vermelho, infravermelho } \\
\text { próximo }\end{array}$ \\
\hline & IRS & $40 \mathrm{~m} / 80 \mathrm{~m}$ & $\begin{array}{l}\text { Pancromática, duas bandas do } \\
\text { Infravermelho de ondas curtas, termal }\end{array}$ \\
\hline & WFI & $64 \mathrm{~m}$ & $\begin{array}{l}\text { Azul, verde, vermelho, infravermelho } \\
\text { próximo }\end{array}$ \\
\hline
\end{tabular}




\begin{tabular}{|c|c|c|c|}
\hline \multirow{3}{*}{ CBERS 04A } & WPM & $2 \mathrm{~m} / 8 \mathrm{~m}$ & \multirow{3}{*}{$\begin{array}{l}\text { Azul, verde, vermelho, infravermelho } \\
\text { próximo }\end{array}$} \\
\hline & MUX & $16 \mathrm{~m}$ & \\
\hline & WFI & $55 \mathrm{~m}$ & \\
\hline \multirow[t]{2}{*}{ LANDSAT 8} & OLI & $30 \mathrm{~m}$ & $\begin{array}{l}\text { Costeira/aerossol, azul, verde, vermelho, } \\
\text { infravermelho próximo, infravermelho de } \\
\text { ondas curtas } 1 \text {, infravermelho de ondas } \\
\text { curtas } 2 \text {, pancromática, Cirrus }\end{array}$ \\
\hline & TIRS & $30 \mathrm{~m} / 100 \mathrm{~m}$ & $\begin{array}{l}\text { Infravermelho termal(TIRS) } 1 \text {, } \\
\text { Infravermelho termal (TIRS) } 2\end{array}$ \\
\hline SENTINEL 2 & MSI & $10 \mathrm{~m} / 20 \mathrm{~m} / 60 \mathrm{~m}$ & $\begin{array}{l}\text { Aerossol, azul, verde, vermelho, borda } \\
\text { do vermelho, infravermelho próximo, } \\
\text { infravermelho curto (Narrow NIR), } \\
\text { vapor d'água, infravermelho de ondas } \\
\text { curtas (Cirrus) e outras duas bandas do } \\
\text { infravermelho de ondas curtas }\end{array}$ \\
\hline $\begin{array}{l}\text { TERRA e AQUA } \\
\text { principal sensor }\end{array}$ & MODIS & $250 \mathrm{~m} / 500 \mathrm{~m} / 1000 \mathrm{~m}$ & $1-2,3-7,8-36$ \\
\hline $\begin{array}{l}\text { IRS } 1 \mathrm{C} \text { e IRC } 1 \mathrm{D} \\
\text { principais sensores }\end{array}$ & LISS - III & $23,6 \mathrm{~m} / 70,7 \mathrm{~m} / 180 \mathrm{~m}$ & $\begin{array}{l}\text { Verde, vermelho, infravermelho próximo, } \\
\text { infravermelho médio }\end{array}$ \\
\hline \multirow{2}{*}{ RESOURCESART-1 } & LISS - III & $23,5 \mathrm{~m}$ & $\begin{array}{l}\text { Verde, vermelho, infravermelho próximo e } \\
\text { infravermelho médio }\end{array}$ \\
\hline & AWiFS & $56 \mathrm{~m}$ & $\begin{array}{l}\text { Verde, vermelho, infravermelho próximo e } \\
\text { infravermelho médio }\end{array}$ \\
\hline
\end{tabular}

Apesar de existirem diversos sensores fornecedores de imagens possíveis de serem utilizadas para mapear as áreas úmidas, deve-se levar em consideração a escala de mapeamento, ou seja, para mapear médias e grandes área úmidas, os produtos da Tabela 1 são úteis; entretanto, para o mapeamento das pequenas áreas úmidas, é necessário um nível mais detalhado. Nunes (2017) propõe a utilização de quatro níveis ou escalas espaciais para mapeamento de áreas úmidas. Do nível de menor detalhe para o de maior detalhe, ou seja, o Nível 1, chamado de Regional e Sub-regional (escala de 1:1.000.000 a 1:500.000); o Nível 2, de sistemas e subsistemas de áreas úmidas (escala entre 1:500.000 a 1:100.000); o Nível 3, capaz de mapear os macro-habitat (escala de 1:100.000 a 1:30.000); e o ultimo nível e mais detalhado, o Nível 4, onde os macro-habitat são detalhados, sendo capazes de reconhecer feições características das fisionomias e espécies (escala de 1:30.000 a 1:500). Lembrando que, de acordo com Nunes da Cunha e Junk (2014), macro-habitat são: unidade da paisagem na planície de inundação com condições hidrológicas e botânicas específicas. Assim, para um mapeamento de áreas úmidas bem-sucedido, necessita-se definir o produto de sensoriamento remoto (imagem), baseado na resolução e no tamanho das áreas úmidas que se pretende mapear, ou seja, para mapeamento de pequenas áreas úmidas, por exemplo, será necessária uma imagem com escala em Nível 4 compatível com sensores de alta resolução e/ou VANTs. 
Tabela 2 - Etapas apropriadas para determinação de dados remotos para inventário de áreas úmidas. Adaptado do apêndice II da Convenção Ramsar (2010) e de Nunes (2017).

Table 2 - Appropriate steps for determining remote data for wetland inventory. Adapted from Appendix II of the Ramsar Convention (2010) and Nunes (2017).

\begin{tabular}{|c|c|}
\hline Etapas & Orientações \\
\hline $\begin{array}{l}\text { 1. Declarar o propósito e o } \\
\text { objetivo. }\end{array}$ & $\begin{array}{l}\text { - Definir explicitamente a finalidade do inventário; por exemplo, definir dados } \\
\text { básicos, tipos de habitat a serem mapeados. }\end{array}$ \\
\hline $\begin{array}{l}\text { 2. Examinar os conhecimentos e } \\
\text { as informações existentes }\end{array}$ & $\begin{array}{l}\text { - Avaliar se os dados disponíveis podem ser aplicados com sucesso, com base } \\
\text { nos objetivos do passo } 1 \text {. Essa avaliação deve ser baseada na relação entre as } \\
\text { características do sensor e a estrutura dos habitat de acordo com a resolução } \\
\text { espacial e espectral do sensor. } \\
\text { * Buscar os dados em instituições de nível internacional, nacional e estadual, } \\
\text { sobre áreas úmidas. }\end{array}$ \\
\hline $\begin{array}{l}\text { 3. Determinar a escala e a } \\
\text { resolução }\end{array}$ & $\begin{array}{l}\text { - Determinar a escala espacial mais adequada para a estrutura do habitat, a } \\
\text { estação de coleta de dados, as características espectrais e a resolução adequada } \\
\text { ao sensor e aos dados já disponíveis. } \\
\text { * No caso de possuir mais campanhas de amostragens, determinar a escala } \\
\text { temporal mais apropriada. }\end{array}$ \\
\hline 4. Escolha apropriada do sensor & $\begin{array}{l}\text { * Avaliar a resolução espacial e espectral de sensores prováveis e assegurar se } \\
\text { possuem as informações necessárias para a questão do passo } 1 \text {. } \\
\text { * Avaliar a necessidade de utilizar vários sensores, como, por exemplo, sensores } \\
\text { de radar, de acordo com as dificuldades de amostragem causadas pela } \\
\text { cobertura de nuvens ou pelo domínio de espécies arbóreas. } \\
\text { * Verificar, para cada sensor, se o tempo de revisita é compatível com o período } \\
\text { de amostragem em campo, se sua aplicação depende de condições sazonais e } \\
\text { o custo e o processamento da imagem estão dentro do orçamento previsto. }\end{array}$ \\
\hline $\begin{array}{l}\text { 5. Metodologia de amostragem em } \\
\text { campo }\end{array}$ & $\begin{array}{l}\text { * Definir uma estratégia de campo. A coleta de dados deverá ser simultânea à } \\
\text { aquisição de dados. } \\
\text { * Determinar quais potenciais problemas poderão influenciar na inferência a } \\
\text { partir dos dados no solo. }\end{array}$ \\
\hline 6. Trade-off & $\begin{array}{l}\text { * Verificar a existência de empresas ou instituições que possam fornecer suporte } \\
\text { às possíveis dificuldades/problemas de utilização dos dados/tecnologias a } \\
\text { serem adquiridos. }\end{array}$ \\
\hline
\end{tabular}

\section{Seleção de categorias e softwares de SIG}

Segundo Câmara e Davis (2001), existem duas formas de representação das características do mundo real em formato computacional: em forma vetorial e forma matricial. Lowry (2006) corrobora com esses formatos citados anteriormente; entretanto, denomina-os como dados discretos e dados contínuos. Assim, comparando os dois autores, pode-se concluir que:

1. nos dados discretos e/ou vetoriais, as representações são associadas a três formas básicas: ponto, linha e polígono. Por exemplo, estradas representadas por linhas; áreas representadas por polígonos; e pontos cotados do terreno por pontos.

2. contínuos, ou também chamados como dados matriciais ou ainda raster, são representações em formato de uma malha quadriculada composta por células com identidades e/ou valores correspondentes à característica a ser representada, como, por exemplo, os 
modelos digitais de elevação, em que cada célula da malha corresponde a um valor de altitude no terreno e possui uma localização (latitude e longitude), ou uma imagem de satélite na qual cada célula contém o respectivo valor de número de brilho (ND).

Dependendo da natureza dos dados disponíveis em cada município, estado e região, devese atentar para a funcionalidade do SIG, ou seja, dependendo da necessidade do usuário, devese escolher o melhor sistema SIG. De acordo com Câmara e Ortiz (1998), devem-se analisar as funcionalidades do SIG em relação a: análise geográfica (ANG), processamento digital de imagens (PDI), modelagem numérica do terreno (MNT), geodesia e fotogrametria (GEO), produção cartográfica (MAP), modelagem de redes (RED) e consulta a banco de dados georreferenciados (BDG). Somente analisando essas funcionalidades torna-se possível selecionar um software de SIG ideal para sua necessidade. Baseado no objetivo do inventário, deve-se selecionar a categoria de SIG mais adequada, ou seja, se existir a necessidade de apenas consulta de dados, um software de SIG com menor complexidade atende o objetivo. Após análise de Câmara e Ortiz (1998) realizada em onze softwares de SIG (ARC/VIEW, ARC/INFO, AutoCad Map, EASI/PACE, ENVI, ERDAS, ER-Mapper, Idrisi, MapInfo,MGE e SPRING), foi possivel observar que a maioria dos softwares não possuem dificuldades em produção cartográfica de mapas e análises geográficas, entretanto, uma minoria apresentam funcionalidades relacionadas à modelagem numérica do terreno $e$ redes. De todos os softwares, apenas dois (ARC/INFO, AutoCad Map) não consegue fazer processamento digital de imagens. Desta maneira, observa-se uma tendência dos sistemas SIG em melhorarem suas funcionalidades, tornando-se cada vez mais completos. Atualmente, o QGIS vem se tornando cada vez mais popular por ser gratuito, e possui todas essas funcionalidades, em forma de módulos, que podem ser instalados pelo usuário, de acordo com a sua necessidade, aprimorando o seu SIG. Mas, se for necessário editar dados, vetoriais ou matriciais, necessita-se de outra categoria de SIG. Lowry (2006) estabeleceu três categorias de software em relação a seu poder de complexidade e custo: i) visualizadores de dados; ii) SIG Desktop; e iii) estações de trabalho (high-end.). Entretanto, existe uma tendência de evolução dos softwares de SIG para atender cada vez mais a um público variado de usuários, permitindo que usuários com experiências e conhecimentos diferentes utilizem o mesmo SIG graças à diversidade de funcionalidade.

\section{Questões-chave para a seleção de dados}

A fim de orientar a escolha dos dados para aplicação em SIG de inventários de AUs, Nunes (2017) descreveu critérios para realização da delimitação e do mapeamento de áreas úmidas via sensoriamento remoto e geoprocessamento. Para atender a esses critérios, foram elaboradas questóes-chave (Tabelas 3 e 4). O conhecimento da escala, projeção e Datum do conjunto de dados (critérios 2 e 3 da Tabela 3 ) pode ser considerado a base para a confiabilidade dos dados, ou seja, para que os dados realmente possam ter confiabilidade da informação representada, como, por exemplo, realizar medidas de distâncias sobre o mapa, todos os dados, vetoriais ou matriciais, utilizados na sua elaboração devem estar no mesmo Datum e na mesma projeção. Isso porque existem diferentes tipos de Datum e projeções e, na maioria das vezes, torna-se necessária a junção de mais de uma camada de dado, que nem sempre está com o mesmo Datum. Dessa maneira, necessitam ser convertidos para o Datum mais atualizado. No Brasil, o Instituto Brasileiro de Geografia e Estatística (IBGE) definiu o SIRGAS 2000 (Sistema de Referência Geocêntrico para as Américas) como o Sistema Geodésico Brasileiro em 2005, ou seja, o Datum no Brasil para ser utilizado é o SIRGAS 2000, mas existem diversos dados que originalmente estão em outro Datum, sendo necessária a conversão. A diferença no terreno de um Datum para outro de um certo local pode variar muito, ou seja, um mesmo local no Datum SIRGAS 2000 pode ter uma diferença mínima para o Datum WGS 84. Assim, muitos usuários podem achar insignificante essa diferença e finalizar o mapa ou a análise em SIG utilizando um mosaico de dados em diferentes Datum. Sobretudo, a melhor forma de se prevenir essa situação é importar o arquivo original e, antes de qualquer outra operação, já o transformar para o Datum de referência que será utilizado no trabalho. 
Além da transformação de Datum, extremamente necessária, alguns dados, como, por exemplo, as imagens de satélite, precisam de correção geométrica. Entretanto, algumas fontes já trazem as imagens corrigidas geometricamente. Um exemplo disso são os níveis de processamento das imagens CBERS. Segundo Paranhos Filho (2016) e Meneses et al. (2012), esses níveis de processamentos são relacionados como processo da correção geométrica puramente matemático e que envolve a aplicação de uma grade corrigida sobre a imagem distorcida, forçando um reposicionamento, por reamostragem pixel a pixel. Sobretudo, como é no caso do satélite CBERS, ainda existem dados fornecidos em formato bruto (nível 0) e, outros, em formato corrigido ou também chamado de ortorretificado (nível 4), sendo necessário observar essa característica do dado antes de utilizá-lo no sistema SIG.

Outra correção indispensável em alguns casos é a correção atmosférica de imagens. As imagens de satélite, em geral, não são fornecidas corrigidas atmosfericamente, com algumas exceções, como, por exemplo, as imagens do satélite Landsat 8 do sensor OLI. Segundo Meneses et al. (2012), esse tipo de correção é mais comprometedor do que os ruídos aleatórios nas imagens. A correção atmosférica não pode ser descartada, principalmente para o caso de análises temporais com uso de imagens de satélite, ou seja, quando há necessidade de comparação entre duas ou mais imagens. Isso porque a radiação que chega ao sensor é atenuada pela atmosfera que sofre variação temporalmente e, portanto, imagens de diferentes anos, meses, dias ou até mesmo horas apresentam atenuações diferentes de atmosfera, o que resulta em imagens com cores diferentes, não devido à mudança real do alvo, mais sim à mudança da atmosfera no momento da aquisição da imagem, o que pode acarretar interpretação equivocada, visto que a área úmida em uma imagem pode ser uma coloração diferente em outra imagem, graças à atenuação atmosférica.

Bernardo (2015) citou e analisou diversos tipos de correções atmosféricas. Os métodos avaliados foram DOS, ELM, FLAASH, ACOLITE e o produto de reflectância do Landsat 8 (denominado como L8SR - Landsat 8 Surface Reflectance), e chegou à conclusão que o FLAASH foi o modelo com menor erro e, portanto, o melhor método; entretanto, a escolha do melhor modelo depende do objetivo do mapeamento que, no caso de Bernardo (2015), foi observar sólidos suspensos em um reservatório de uma usina hidroelétrica. Por fim, ressalta-se que, independente do objetivo, no caso de utilizar imagens de satélite para análises temporais, devem-se utilizar dados corrigidos atmosfericamente.

Tabela 3 - Critérios e questões-chave para a seleção de dados de sistemas de informações geográficas. Table 3 - Criteria and key questions for selection of data from geographic information systems.

\begin{tabular}{|c|c|}
\hline Critérios de seleção de dados SIG & Questões-chave \\
\hline 1. Fundamentação & $\begin{array}{l}\text { * O que realmente quero demonstrar? } \\
\text { * Preciso de quais conjuntos de dados específicos? } \\
\text { Existem outros conjuntos de dados possíveis de representar o mesmo } \\
\text { recurso mais efetivamente? }\end{array}$ \\
\hline 2. Escala & $\begin{array}{l}\text { * A escala é apropriada para o nível de estudo realizado? } \\
\text { * A escala dos dados é compatível, na utilização em conjunto, dentro } \\
\text { do sistema SIG? }\end{array}$ \\
\hline $\begin{array}{l}\text { 3. Projeção e Datum do conjunto de } \\
\text { dados }\end{array}$ & $\begin{array}{l}\text { * Em que projeção e Datum o projeto de SIG e o conjunto de dados } \\
\text { remotos foi criado? } \\
\text { * A projeção de Datum do conjunto de dados selecionados e do projeto } \\
\text { de SIG são compatíveis? }\end{array}$ \\
\hline
\end{tabular}




\begin{tabular}{|c|c|}
\hline 4. Confiabilidade & $\begin{array}{l}\text { * Quais métodos foram utilizados na criação do conjunto de dados? } \\
\text { * Foi utilizada metodologia reconhecida e/ou corretamente documentada } \\
\text { na criação/compilação dos dados? }\end{array}$ \\
\hline 5. Integridade & $\begin{array}{l}\text { - O conjunto de dados está espacialmente e/ou topologicamente } \\
\text { completo? } \\
\text { Existe um registro de metadados para o conjunto de dados? }\end{array}$ \\
\hline $\begin{array}{l}\text { 6. Direitos de autoria, distribuição e } \\
\text { questões jurídicas }\end{array}$ & $\begin{array}{l}\text { * O direito de reprodução de cada conjunto de dados do custodiante } \\
\text { dos dados originais foi estabelecido? } \\
\text { * É necessário estabelecer um contrato de licença, com o custodiante } \\
\text { dos dados originais, para o uso e distribuição dos dados que serão } \\
\text { utilizados? }\end{array}$ \\
\hline 7. Custo & $\begin{array}{l}\text { * Serão utilizados dados gratuitos ou pagos? } \\
\text { - Os dados possuem alguma limitação significativa (por exemplo, } \\
\text { escala) para atender ao objetivo do trabalho? }\end{array}$ \\
\hline
\end{tabular}

\section{Questões-chave de seleção de softwares de SIG}

Da mesma forma que os dados de SIG, foram criadas questões-chave para a seleção de softwares de SIG (Tabela 4).

Em relação ao custo do software, é preciso saber se o treinamento exigido para operar corretamente o software tem algum custo, isso porque, segundo Câmara e Davis (2001), pode ser mais vantajoso adquirir o software com custo mais elevado inicialmente, que envolva um treinamento de baixo custo. Sendo assim, um preço elevado não é necessariamente sinônimo de um produto útil. Deve-se avaliar, caso a caso, o custo-benefício do software e seu treinamento, tendo sempre como base o propósito/objetivo específico do uso para que o objetivo possa ser alcançado utilizando-se o menor custo possível.

O terceiro critério, descrito na Tabela 4, a compatibilidade, apresenta uma tendência de ser cada vez um problema menor, ou seja, atualmente e no futuro com a capacidade dos programas de ler e integrar dados de vários formatos, a questão da compatibilidade tende a ser negligenciada.

Apesar de existirem técnicas de processamento e gestão dos dados bem desenvolvidas, a necessidade de verificação em campo das informações mapeadas é indispensável (Lowry 2006, Ramsar 2010, Aronoff 1989, Câmara \& Davis 2001).

Tabela 4 - Critérios e questões-chave de seleção de softwares de sistemas de informações geográficas. Table 4 - Criteria and key selection issues for geographic information systems software.

\begin{tabular}{|c|c|}
\hline $\begin{array}{c}\text { Critérios de seleção de software/ } \\
\text { programas de SIG }\end{array}$ & Questões-chave \\
\hline 1. Requisitos/componentes do software & $\begin{array}{l}\text { - Que tipo de atividade está planejando realizar no software (visualizar, } \\
\text { criar, editar, atualizar os dados)? }\end{array}$ \\
\hline 2. Custo & $\begin{array}{l}\text { * Quais recursos/capacidade o software oferece? } \\
\text { * O software pode ser usado para o objetivo pretendido de aplicação? } \\
\text { * O programa oferece espaço para expansão/crescimento, permitindo } \\
\text { análises adicionais ou melhoradas no futuro? } \\
\text { * Qual nível de apoio à venda, instalação e operação o fabricante } \\
\text { fornece? } \\
\text { * Existe custo de treinamento para uso do software? }\end{array}$ \\
\hline
\end{tabular}


3. Compatibilidade com outros programas

. Capacidade de hardware

5. Facilidade de utilização
- O software tem capacidade de salvar, importar e exportar arquivos de formatos diversos?

* A capacidade do computador é compatível com o máximo de uso possível de dados a serem utilizados (espaço no disco rígido, velocidade e capacidade de processamento)?

* Até que ponto é fácil para pessoas, com diferentes graus de experiências em computadores e SIG, dominar e realizar as tarefas no software?

* É necessário treinamento?

\section{Conclusão}

Para auxiliar na escolha dos dados de sensoriamento remoto e SIG no inventário de AUs, foram definidos, neste trabalho, os passos integrados e as orientações para garantir a realização de um inventário de AUs.

O trabalho contribuiu para a Estratégia para Implementação da Convenção de Ramsar e dos Sítios Ramsar no Brasil, através de procedimentos comuns baseados em diretrizes da Convenção Ramsar (Ramsar Convention Secretariat (2010). Wetland inventory e Relatório técnico Lowry, J. (2006)), esclarecendo informações sobre o uso de dados remotos e softwares de SIG como ferramentas a serem utilizadas em inventários, avaliações e monitoramentos de AUs. As informações compiladas fornecem instrumentos para capacitação dos gestores em suas rotinas de manejo e seus planejamentos, viabilizando a troca de experiência entre a Rede Nacional de Gestores de Sítios e comunidade de aprendizagem para AUs com ferramentas (dados remotos e SIG) para tomada de decisões das iniciativas brasileiras voltadas ao monitoramento das AUs.

\section{Referências bibliográficas}

Aronoff, S. 1989. Geographic information systems: a management perspective. WDL Publications. 294p.

Bernardo, N.M.R. 2015. Análise do potencial do sensor OLI (Operational Land Imager) a bordo do Landsat-8 em estimar a concentração de sólidos totais em suspensão no reservatório de Barra Bonita. Dissertação (Mestrado em Ciências Cartográficas). Universidade Estadual Paulista, Faculdade de Ciências e Tecnologia. 117p.

Câmara, G. \& Ortiz, M.J. 1998. Sistemas de informação geográfica para aplicações ambientais e cadastrais: uma visão geral. p. 59-82. In: Congresso Brasileiro de Engenharia Agrícola.

Câmara, G. \& Davis, C. 2001. Arquitetura de Sistemas de Informação Geográfica. In: Câmara, G.; Davis, C. \& Monteiro, A.M. (org.), Introdução à Ciência da Geoinformação. < http://www.dpi.inpe.br/gilberto/ livro/introd/>. (Acesso em 10/10/2017).

Davidson, N.C. \& Finlayson, C.M. 2007. Earth observation for wetland inventory, assessment and monitoring. Aquatic Conservation: Marine and Freshwater Ecosystems, 17(3): 219-228.

Lowry, J. (2006). Low-cost GIS software and data for wetland inventory, assessment and monitoring. Ramsar, Australia 2, 2006. Ramsar Technical Reports n. 2 (Ramsar Convention Secretariat Gland, Switzerland). $32 \mathrm{p}$.

Meneses, P.R. et al. 2012. Introdução ao processamento de imagens de sensoriamento remoto. Universidade de Brasilia.

Nogueira, R.E. 2008. Cartografia: representação, comunicação e visualização de dados espaciais. Ed. UFSC. 170p. 
Nunes, G.M. 2017. Marco metodológicos para a delimitação e mapeamento de áreas úmidas in: marcos referenciais para a lei federal do Pantanal e gestão de outras áreas úmidas. 1 ed. Carlini e Caniato e Edufmt, v.1, p. 45-53.

Nunes da Cunha, C. \& Junk, W.J. 2014. A Classificação dos Macrohabitats do Pantanal Mato-grossense. p. 77-122. In: Nunes da Cunha, C; Piedade, M.T.F. \& Junk, W.J. (orgs.). Classificação e Delineamento das Áreas Úmidas Brasileiras e de seus Macrohabitats. EdUFMT.

Paranhos Filho, A.C.; Mioto, C.L. \& Junior, J.M. 2016 (ed.). Geotecnologias em aplicações ambientais. Editora UFMS. 383p.

Ramsar Convention Secretariat. 2010. Wetland inventory: a Ramsar framework for wetland inventory and ecological character description. 4 ed. v.15. Ramsar Convention Secretariat. 88p.

Rebelo, L.M.; Finlayson, C.M. \& Nagabhatla, N. 2009. Remote sensing and GIS for wetland inventory, mapping and change analysis. Journal of Environmental Management, 90(7): 2144-2153.

Biodiversidade Brasileira - BioBrasil.

Número temático: Diagnóstico e manejo de áreas úmidas em áreas protegidas

$$
\text { n. 2, } 2019
$$

http://www.icmbio.gov.br/revistaeletronica/index.php/BioBR

Biodiversidade Brasileira é uma publicação eletrônica científica do Instituto Chico Mendes de Conservação da Biodiversidade (ICMBio) que tem como objetivo fomentar a discussão e a disseminação de experiências em conservação e manejo, com foco em unidades de conservação e espécies ameaçadas.

ISSN: 2236-2886 